\title{
Systemic inflammatory markers and sources of social support among older adults in the Memory Research Unit cohort
}

Journal of Health Psychology 2019, Vol. 24(3) 397-406 (C) The Author(s) 2016 Article reuse guidelines: sagepub.com/journals-permissions DOI: $10.1|77 /| 35910531667633$ | journals.sagepub.com/home/hpq @SAGE

\author{
Joanna McHugh Power',2, Sile Carney', \\ Caoimhe Hannigan', Sabina Brennan', \\ Hannah Wolfe', Marina Lynch', Frank Kee ${ }^{2}$ \\ and Brian Lawlor'
}

\begin{abstract}
Potential associations between systemic inflammation and social support received by a sample of 120 older adults were examined here. Inflammatory markers, cognitive function, social support and psychosocial wellbeing were evaluated. A structural equation modelling approach was used to analyse the data. The model was a good fit $\left(\chi_{108}^{2}=256.13, p<0.001\right.$; comparative fit index $=0.973$; Tucker-Lewis Index $=0.962$; root mean square error of approximation $=0.02 \mathrm{I}$; standardised root mean-square residual $=0.074$ ). Chemokine levels were associated with increased age $(\beta=0.276)$, receipt of less social support from friends $(\beta=-0.256)$ and body mass index $(\beta=-0.256)$. Results are discussed in relation to social signal transduction theory.
\end{abstract}

\section{Keywords}

age, health psychology, protective factors, quantitative methods

\section{Introduction}

Social isolation and social support are correlated with health and mortality (Berkman and Syme, 1979; Cohen, 1988; Holt-Lunstad et al., 2015; House et al., 1982). The mechanisms underlying this relationship include cardiovascular functioning (Shankar et al., 2011), hormonal functioning (Knox and Uvnäs-Moberg, 1998), hypothalamic-pituitary-adrenal (HPA) axis activation (Eisenberger and Cole, 2012), neurogenesis (Stranahan et al., 2006), health behaviours and stress (Cacioppo and Hawkley, 2009). Social functioning and inflammatory processes are also correlated. According to social signal transduction theory, this is due to the effect of perceived social threat on both physiological stress and inflammatory responses (Slavich and Irwin, 2014; Slavich et al., 2010a, 2010b).

\footnotetext{
'Trinity College, University of Dublin, Republic of Ireland ${ }^{2}$ Queen's University Belfast, UK

\section{Corresponding author:}

Joanna McHugh Power, Centre for Public Health, Institute of Clinical Sciences, Block B, Queen's University Belfast, Royal Victoria Hospital, Grosvenor Road, Belfast BTI2 6BJ, UK.

Email: J.McHugh@qub.ac.uk
} 
Social signal transduction theory predicts that inflammatory responses are driven by the experience of social threat, such as that may occur when an individual's social support levels are low. In support of this prediction, structural social factors such as number of social ties (Cohen et al., 1997; Ford et al., 2006) and social isolation (Loucks et al., 2006; Yang et al., 2014) are found to be related to inflammatory responses. However, these structural factors may be contextual (rather than causal) factors for inflammation, since they represent the context within which social support may be provided (Penwell and Larkin, 2009).

More proximate social factors are also related to inflammatory processes. Perception of high levels of social support has been shown to relate to lower levels of C-reactive protein (CRP) in adults (Runsten et al., 2014) and lower levels of interleukin-6 (IL-6) among survivors of breast cancer (Hughes et al., 2014). Instrumental support has also been shown to be associated with lower levels of systemic inflammatory activity (Costanzo et al., 2005).

Thoits has defined two sources of support: one that provided by significant others, such as spouses and family members, and the other that provided by experientially similar others, including friends and others whose experiences overlap with those of the recipient. According to Thoits (2011), experientially similar others provide superior assistance in coping, relative to significant others. Few studies have examined whether Thoits' distinction is reflected in physical outcomes such as inflammatory markers. While social support is often measured as a uni-dimensional construct, it has been argued that support from different sources may have different effects on health (DuPertuis et al., 2001). Furthermore, social support from family is found to be more closely related to cardiovascular factors than social support from other sources (Uchino et al., 1996). Other evidence suggests that the experience of loneliness in later life is more likely to be due to insufficient contact with friends or neighbours, than with family members (Pinquart and Sorensen, 2001). Nakata et al. (2014) investigated the relationship between different sources of support and inflammatory markers among a sample of working adults. Nakata found that social support from employment supervisors (but not from co-workers, friends or family) was inversely related to circulating IL-6 concentrations. It is possible that social support may be differentially related to inflammation on the basis of the source of the support. While Nakata's research focused on a working population, we wanted to investigate whether sources of social support relate to inflammation among an older sample, including those who are retired, and for whom work-based social support is unlikely.

Lubben and Gironda (2004) define three pertinent sources of social support in later life: neighbours, friends and family members. We used this classification of social support to investigate whether there is an association between inflammation and support received from experientially similar others (in this case, friends) as opposed to other sources of support, such as neighbour or family-derived social support.

\section{Methods}

\section{Design}

A sample of adults was recruited from an ongoing observational cohort study at the Memory Research Unit at Trinity College Dublin. This study recruited almost 1000 participants at baseline and conducts follow-up assessments of biopsychosocial and cognitive wellbeing every 2 years. During Wave 2, which commenced during 2014, 120 participants were selected according to a convenience sampling protocol to participate in a sub-study, the results of which pertain to the current investigation.

\section{Participants}

Of the original cohort, 120 participants were invited to take part in an additional sub-study, which involved venepuncture and the collection of $10 \mathrm{~mL}$ blood serum. The first 120 participants returning to the original cohort study for followup were invited to take part in this sub-study. Of 
the first 120 participants invited to participate, all 120 participants consented in written form. These participants also took part in the overall study's standard biopsychosocial and cognitive evaluation (Hannigan, 2015). The original study was approved by the School of Psychology Ethics Committee, and this sub-study was approved by the Health Sciences Research Ethics Committee, both at Trinity College Dublin. Participants were recruited to the sub-study contingent on the following exclusion criteria: history of stroke, neurological problems, psychiatric illness or substance abuse, uncontrolled hypertension, Parkinson's disease, brain surgery, learning disability, brain tumour, multiple sclerosis, cerebral palsy, Huntington's disease, kidney disease (evaluated using the Christensen Health Screen Questionnaire; Christensen et al., 1992), epilepsy, current use of anti-psychotic or antiepileptic medications, self-reported significant memory problems or dementia, or sensory issues that would preclude neuropsychological assessment. Inclusion criteria were that the participant was aged over 50 years, resident in Ireland, fluent in English to a degree that they were able to complete cognitive assessments and able to travel independently to the research institute to participate in the research study. To take part in this sub-study, participants also had to indicate that they had not suffered from any viral or bacterial illness in the previous 2 weeks. Of the initial 120 participants, four were excluded: two had lupus and two had insufficient levels of serum taken from the venepuncture procedure. Of the remaining 116, 76 were females (mean age $=65.85$ years, standard deviation $(\mathrm{SD})=6.59$, age range $=51-81$ years)

\section{Procedure}

As well as participating in the original biopsychosocial component of the research, participants in this study also gave a blood sample. During venepuncture, a trained phlebotomist collected $10 \mathrm{~mL}$ of serum from each participant. Serum samples were centrifuged at $2000 \mathrm{r} / \mathrm{min}$ for 10 minutes, to remove residual cells and debris. Supernatants were stored at $-80^{\circ}$ until they were analysed for inflammatory biomarkers using Meso Scale Discovery technology. Assay kits were run in duplicate according to the manufacturer's instructions. A V-Plex validated 30-Plex plate (catalogue number K15054D-1), detecting the presence of inflammatory and anti-inflammatory cytokines, chemokines and other pro-inflammatory markers, was used. Plates were incubated with samples and standards for 2 hours, washed, and detection antibody added for 2 hours before the plate was read. This detected a total of 30 biomarkers (Eotaxin, Eotaxin 3, vascular endothelial growth factor (VEGF), granulocyte-macrophage colony-stimulating factor (GM-CSF), interferongamma (IFN $\gamma$ ), IL10, IL12/IL-23p40, IL-12p70, IL13, IL15, IL16, IL17A, IL1 $\alpha$, IL1 $\beta$, IL2, IL4, IL5, IL6, IL7, IL8, IL8(HA), interferon gammainduced protein 10 (IP10), mast cell protease 1 (MCP1), MCP4, macrophage-derived chemokine (MDC), macrophage inflammatory protein 1 -alpha (MIP1 $\alpha$ ), MIP1 $\beta$, thymus- and activationregulated chemokine (TARC), tumour necrosis factor-alpha (TNF $\alpha)$, TNF $\beta)$. Problematic missingness (over 80\%) affected the Eotaxin 3 and the IL1 $\beta$ markers, and values of these markers, and of any the other values of markers less than the lower limit of detection, were thus excluded from further analyses.

\section{Measures}

Covariates (age, gender, number of years in education, height and weight) were collected from all participants. Depression was measured using the well-validated and reliable 20-item Centre for Epidemiological Studies Depression Scale (CESD20; Radloff, 1977). Reliability was high in the current sample $(\alpha=0.81)$. Social support was evaluated using an 18-item version of the Lubben Social Network Scale (Lubben and Gironda, 2004). This scale evaluates social support from friends, neighbours and family, with higher scores indicating higher levels of support. The Lubben Scale gave a high reliability in the current sample $(\alpha=0.85)$. A comorbidity count was created from the Health Screening Questionnaire (Christensen et al., 1992) from self-reports of histories of the following illnesses: cancer other than skin cancer, 
Table I. Descriptive statistics and psychometrics (where applicable) for $n=1 \mid 6$ participants in the Memory Research Unit.

\begin{tabular}{llcc}
\hline & Mean/percentage & SD & $\begin{array}{c}\text { Cronbach's } \\
\text { alpha }\end{array}$ \\
\hline Age & 65.81 & 6.63 & \\
Sex & $66 \%$ females; 33\% males & & \\
Years in education & 14.68 & 3.21 & 0.81 \\
CESD & 5.14 & 5.3 & 0.85 \\
LSNS total & 51.77 & 12.01 & 0.76 \\
LSNS family & 21.52 & 4.63 & 0.82 \\
LSNS neighbours & 12.37 & 5.94 & 0.84 \\
LSNS friends & 17.58 & 5.61 & \\
BMI & 26.24 & 3.71 & \\
\hline
\end{tabular}

Data were collected during 2015.

LSNS: Lubben Social Network Scale; CESD: Centre for Epidemiological Studies Depression Scale; BMI: body mass index.

use of home oxygen, heart surgery, diabetes requiring insulin, encephalitis or meningitis, heart attack and seizures. Of the 116 individuals, 84 per cent had no comorbidity, 13 per cent had one, 2 per cent had two and 1 per cent had three comorbidities.

\section{Data analysis}

To reduce the dimensionality and the chance of Type 1 errors for the main hypothesis tests, an initial exploratory factor analysis was run to evaluate the inflammatory markers. Since three panels were used for the purposes of this study, nominally cytokines, chemokines and proinflammatory markers, three factors were specified in the model. A principal axis extraction method with pro-max rotation was used. A parallel analysis was also used to confirm the number of suitable factors. Upon conducting the exploratory factor analysis, factor loadings were examined to identify items not clearly loading on any single factor, defined here as a factor loading of less than 0.3 . Within the lavaan package in R (Rosseel, 2012), a confirmatory factor analysis was then used to confirm the factors arising from the exploratory factor analysis. Finally, a structural equation model was created to evaluate the relationship between these factors and loneliness and social support, controlling for covariates.

\section{Results}

Sample characteristics are laid out in Table 1.

\section{Exploratory factor analysis}

First, a Kaplan-Meier-Olkin (KMO) measure of sampling adequacy was calculated to evaluate the suitability of the data for factor analysis and was found to be adequate $(\mathrm{KMO}=0.47)$. Bartlett's test of sphericity was also conducted and corroborated the suitability of the data for factor analysis $\left(\chi_{136}^{2}=172.1, p<0.001\right.$; objective function $=1.58$; see Table 2 ). We examined the patterns of factor loadings for each principal component in turn. For principal component 2, the markers Eotaxin, TARC, IP10, MDC1 and MCP4 all had high factor loadings and little cross loadings. This component is hereafter referred to as the chemokines (although we recognise that MCP1, MIP $1 \alpha$ and MIP1 $\beta$ are also chemokines). For principal component 3 , the markers TNF $\alpha$, IL7, IL16 and IL17 all loaded well without cross loading. This component was hereafter referred to as the cytokines. For principal component 1 , however, there were problematic loadings such that only IFN $\gamma$ belonged to the third family of markers, the so-called 'other pro-inflammatory markers'. Thus, only the chemokines and cytokines were included as latent factors in the following analyses. 
Table 2. Total variance explained by the exploratory factor analysis conducted on the inflammatory marker data $(n=\mid 18)$, and factor loadings on each of the three factors for all inflammatory markers (principal axis extraction methods with pro-max rotation used).

\begin{tabular}{llllll}
\hline Component & $\begin{array}{l}\text { Squared Sum } \\
\text { of loadings }\end{array}$ & $\begin{array}{l}\text { Proportion } \\
\text { variance }\end{array}$ & $\begin{array}{l}\text { Cumulative } \\
\text { variance }\end{array}$ & $\begin{array}{l}\text { Proportion } \\
\text { explained }\end{array}$ & $\begin{array}{l}\text { Cumulative } \\
\text { proportions }\end{array}$ \\
\hline 2 & 2.37 & 0.16 & 0.16 & 0.38 & 0.38 \\
I & 2 & 0.13 & 0.29 & 0.32 & 0.7 \\
3 & 1.88 & 0.13 & 0.42 & 0.30 & I \\
\hline Item & PC2 & PCI & PC3 & Communality $\left(h^{2}\right)$ & Uniqueness $\left(u^{2}\right)$ \\
\hline Eotaxin & 0.59 & -0.35 & 0.03 & 0.37 & 0.63 \\
MIPI $\beta$ & 0.11 & 0.18 & 0.28 & 0.20 & 0.80 \\
TARC & 0.77 & 0.1 & -0.25 & 0.56 & 0.44 \\
IPI0 & 0.20 & 0.16 & 0.19 & 0.17 & 0.83 \\
MIPI $\alpha$ & 0.20 & -0.06 & 0.22 & 0.11 & 0.89 \\
MCPI & 0.40 & -0.16 & 0.53 & 0.51 & 0.49 \\
MDCI & 0.63 & 0.22 & -0.12 & 0.47 & 0.53 \\
MCP4 & 0.85 & -0.11 & -0.12 & 0.63 & 0.37 \\
IFN $\gamma$ & -0.21 & -0.25 & 0.88 & 0.61 & 0.39 \\
TNF $\alpha$ & 0.09 & 0.49 & 0.11 & 0.33 & 0.67 \\
IL7 & 0.19 & 0.29 & 0.16 & 0.24 & 0.76 \\
ILI5 & -0.34 & 0.12 & 0.71 & 0.5 & 0.50 \\
ILI6 & -0.1 & 0.95 & -0.31 & 0.75 & 0.25 \\
ILI7 & -0.02 & 0.76 & 0.06 & 0.61 & 0.39 \\
VEGF & 0.00 & 0.02 & 0.44 & 0.2 & 0.8 \\
\hline
\end{tabular}

MIPI: macrophage inflammatory protein I; TARC: thymus- and activation-regulated chemokine; IPI0: interferon gamma-induced protein I0; MCP: mast cell protease I; MDC: macrophage-derived chemokine; IFN $\gamma$ : interferon-gamma; TNF $\alpha$-tumour necrosis factor-alpha; IL: interleukin; VEGF: vascular endothelial growth factor.

\section{Structural equation model}

Using the lavaan package (Rosseel, 2012), a structural equation model with the maximum likelihood estimator and a full information maximum likelihood approach to missingness was used to evaluate the relationship between social support sources, loneliness and the two inflammatory factors, cytokines and chemokines, as outcomes. Age, sex, body mass index (BMI), CESD and comorbidity count were all included as covariates in the model. Factors were not set to be orthogonal. The model converged normally after 97 iterations, and had acceptable fit $\left(\chi_{108}^{2}=256.13, p<0.001\right.$; comparative fit index $(\mathrm{CFI})=0.973$; Tucker-Lewis Index $(\mathrm{TLI})=0.962$; root mean square error of approximation $($ RMSEA $)=0.021 \quad(0.000,0.058) ;$ standardised root mean-square residual $(\mathrm{SRMR})=0.074)$. The measurement component of the model indicated that all items loaded well onto the cytokine and chemokine factors (see Table 3). Increased age, lower BMI and lower levels of social support from friends were associated with higher levels of chemokine (see Table 3); no variables were significantly related to cytokine levels.

\section{Discussion}

The current findings suggest that social support given by friends would be associated with systemic inflammation, in keeping with Thoits' (2011) differentiation between types of social support. Other research also shows that social support distinguished by source can impact differentially on wellbeing in later life (DuPertuis et al., 2001). Instrumental support provided for older adults by family members may be more 
Table 3. Structural equation model with cytokine and chemokine as outcomes, with social support (family = support from family; friends = support from friends; neighbours = support from neighbours) as predictors, and age, sex, body mass index, depression and comorbidity count as covariates (standardised estimates (Std. Est): for the measurement model, these are equivalent to factor loadings; for the structural model, these are equivalent to beta coefficients).

\begin{tabular}{|c|c|c|c|c|c|c|}
\hline & Estimate & Std. error & $Z$ & $p$ & Std. Est & $R^{2}$ \\
\hline \multicolumn{7}{|c|}{ Measurement model } \\
\hline Chemokine & & & & & & 0.254 \\
\hline Eotaxin & 0.253 & 0.087 & 2.92 & 0.004 & 0.295 & 0.087 \\
\hline TARC & 0.675 & 0.092 & 7.365 & 0.000 & 0.778 & 0.606 \\
\hline IPIO & 0.261 & 0.123 & 2.125 & 0.034 & 0.298 & 0.089 \\
\hline $\mathrm{MDCl}$ & 0.489 & 0.086 & 5.67 & 0.000 & 0.568 & 0.323 \\
\hline MCP4 & 0.516 & 0.090 & 5.727 & 0.000 & 0.595 & 0.354 \\
\hline Cytokine & & & & & & 0.183 \\
\hline TNF $\alpha$ & 0.624 & 0.140 & 4.462 & 0.000 & 0.689 & 0.474 \\
\hline IL7 & 0.400 & 0.097 & 4.116 & 0.000 & 0.445 & 0.198 \\
\hline ILI6 & 0.296 & 0.100 & 2.949 & 0.003 & 0.331 & 0.110 \\
\hline ILI7 & 0.330 & 0.113 & 2.930 & 0.003 & 0.385 & 0.148 \\
\hline \multicolumn{7}{|l|}{ Structural model } \\
\hline \multicolumn{7}{|l|}{ Chemokine } \\
\hline Age & 0.048 & 0.019 & 2.588 & 0.010 & 0.276 & \\
\hline Sex & -0.000 & 0.064 & -0.007 & 0.995 & -0.001 & \\
\hline BMI & -0.081 & 0.034 & -2.396 & 0.017 & -0.256 & \\
\hline Family & 0.009 & 0.029 & 0.327 & 0.744 & 0.038 & \\
\hline Neighbour & 0.023 & 0.023 & 1.023 & 0.306 & 0.120 & \\
\hline Friends & -0.054 & 0.025 & -2.116 & 0.034 & -0.259 & \\
\hline Depression & 0.045 & 0.023 & 1.910 & 0.056 & 0.204 & \\
\hline Comorbidity & 0.219 & 0.246 & 0.890 & 0.374 & 0.093 & \\
\hline \multicolumn{7}{|l|}{ Cytokine } \\
\hline Age & 0.027 & 0.021 & 1.286 & 0.141 & 0.181 & \\
\hline Sex & 0.092 & 0.073 & 1.262 & 0.190 & 0.163 & \\
\hline BMI & 0.054 & 0.037 & 1.432 & 0.161 & 0.174 & \\
\hline Family & 0.022 & 0.032 & 1.691 & 0.469 & 0.096 & \\
\hline Neighbour & 0.015 & 0.027 & 0.570 & 0.582 & 0.079 & \\
\hline Friends & -0.036 & 0.028 & -1.286 & 0.189 & -0.187 & \\
\hline Depression & 0.016 & 0.026 & 0.607 & 0.551 & 0.074 & \\
\hline Comorbidity & 0.362 & 0.286 & 1.264 & 0.224 & 0.154 & \\
\hline
\end{tabular}

TARC: thymus- and activation-regulated chemokine; IPI0: interferon gamma-induced protein I0; MCP4: mast cell protease 4; MDCI: macrophage-derived chemokine I; TNF $\alpha$-tumour necrosis factor-alpha; IL: interleukin; BMI: body mass index.

predictive of physical wellbeing (Friedman, 1993), than is the case for support from friends, which may be more likely to improve mood (Lee and Ishii-Kuntz, 1987). Social support from family may increase both positive and negative affect, while social support from friends may increase positive affect and decrease negative affect among older adults (Huxhold et al., 2014). Similarly, Li et al. (2014) found that among older adults, social support from friends was associated with increased positive affect, but social support from family was not. These findings make sense in relation to Weiss' (1974) functional specificity model of relationships, which 
states that different relationships perform different functions.

Social support from friends was related to lower levels of circulating chemokines, but no such relationship was found with cytokines. This finding likely reflects the healthy nature of the sample, rendering them less likely to have high levels of circulating cytokines, which are associated with more inflammation, than levels of chemokines, which could be more useful for detecting subtle differences in inflammation across an otherwise healthy sample. A negative association between BMI and chemokines was found, such that lower BMI was associated with higher levels of circulating chemokines, which is contrary to what is typically found in the literature, since obesity is described as a state of low-grade chronic inflammation (Xu et al., 2015). However, BMI was included in the model as a linear term, while in reality it likely has a non-linear relationship with inflammation, since both low and high levels of BMI are generally associated with negative health outcomes. This finding makes sense in relation to a previous meta-analysis, which found a role for midlife, but not later life, BMI, in predicting later-life dementia (Anstey et al., 2011).

The current findings accord with previously published literature suggesting a relationship between social support and inflammatory processes (Costanzo et al., 2005; Runsten et al., 2014), and also partly corroborate the social signal transduction theory (Slavich et al., 2010b), which predicts associations between social support levels and inflammation. However, results did not indicate a relationship between all subtypes of social support and inflammation. The theory also predicts relationships between depression and inflammatory processes, which, while approaching significance, were not borne out by the data. There was a trend such that higher levels of chemokines were related to higher scores on the CESD, but this relationship did not reach statistical significance. The generally healthy status of the sample may be pertinent here, such that associations may only become apparent in more severely affected individuals. Future research focusing on older participants who satisfy clinical diagnostic criteria could interrogate the potential relationships among social support, depression and inflammatory processes more stringently. Other facets of proximal social interaction could also increase the understanding of the relationship between social support and inflammation. Lower levels of social participation are associated with higher levels of plasma concentrations of fibrinogen among men (Helminen et al., 1997), and social activities reported among females with cancer are inversely related to plasma concentrations of TNF $\alpha$ (Marucha et al., 2005). An investigation of social participation and engagement in social activities and their association with inflammatory processes in the healthy ageing population could increase the understanding and inform intervention development and policy and practice.

A methodological limitation of this study is that the design was cross sectional, precluding comment on the potential causal nature of the relationship between inflammation and social support. Thus, as well as considering the social signal transduction theory as a possible explanation of the finding, the sickness behaviour theory of Dantzer (2001), which predicts that inflammation drives social withdrawal, may be a viable alternative. Future research utilising longitudinal panel data would be required to evaluate how well the data fit either of these theoretical alternatives. Atop this, the sample size was small, particularly relative to the crosssectional nature of the study design. This limitation means that the study findings might not be representative of the general ageing population. This caveat must be considered in relation to the current findings and could potentially be addressed in future studies with larger sample sizes. Another possibility for future research would be to use a stratified sample and purposively recruit individuals with high, medium and low levels of social support from different sources, in order to compare their levels of systemic inflammation.

The current analysis also had some methodological strengths. The majority of previously published literature investigating the link between social support and inflammation has 
focussed on a small number of inflammatory markers (Costanzo et al., 2005; Hughes et al., 2014; Runsten et al., 2014). This study utilised a large number of inflammatory markers, allowing the creation of a latent construct of systemic inflammation. The measurement model indicates that this is an acceptable methodology when evaluating systemic inflammation, since the data point towards the existence of a latent 'systemic inflammation' construct. Furthermore, structural equation modelling was used to model the hypothesised relationship between social support and inflammation among older adults. Structural equation modelling is a flexible and powerful approach that can incorporate the simultaneous modelling of mediating pathways. While controversy exists surrounding sample sizes appropriate for use in structural equation modelling (Wolf et al., 2013), this study's relatively small sample size of 116 meets previously published guidelines for conducting such analyses (Bentler and Chou, 1987; Boomsma, 1982). Furthermore, it has been argued that power within structural equation modelling is determined by more than sample size, such as the degree of misspecification and variance accounted for by the latent constructs within the manifest variables (Tomarken and Waller, 2003). To address the potential power issues, several indices of fit have here been reported, including the SRMR, which measure the residual matrix of the observed and implied variances and covariances, and as such can detect misspecification. Variances accounted for by the latent constructs within the manifest variables were also reported.

This study provides evidence that the source of social support may matter when considering physical health. This finding makes sense when considering the assertions by DuPertuis et al. (2001) that social support has different effects depending on its source (Huxhold et al., 2014; Lee and Ishii-Kuntz, 1987; Weiss, 1974). Specifically, it was found social support received from friends is associated with lower levels of inflammatory chemokines, which may corroborate Thoits' (2011) proposal that experientially similar others offer different forms of support from the more obligatory sources of support such as is provided by family and neighbours. Results have implications for future research. Further analyses are required to investigate the mechanisms through which social support from friends relates to physical health and inflammatory processes. The study sample was free from significant burdens of illness, but it is possible that in a more representative older sample, inflammation related to poor physical health may relate to social support because of its association with chronic illness or disease. Further research is warranted before policy recommendations regarding sources of social support in later life can be made.

\section{Acknowledgements}

The authors wish to thank all the participants involved in the research study and the research assistants who were involved in collecting data. The authors also wish to acknowledge the Division of Ageing Research and Development at the Institute of Public Health, formerly the Centre for Ageing Research and Development in Ireland, who funded a fellowship in Leadership in Ageing Research for the first author (J.M.P.), through which the study was funded.

\section{Declaration of Conflicting Interests}

The author(s) declared no potential conflicts of interest with respect to the research, authorship and/or publication of this article.

\section{Funding}

The author(s) disclosed receipt of the following financial support for the research, authorship, and/or publication of this article: The original study was funded in part by Atlantic Philanthropies. The substudy analysed in the current manuscript was funded by a fellowship received by the first author (J.M.P) from the Division of Ageing Research and Development at the Institute of Public Health in Ireland, formerly the Centre for Ageing Research and Development in Ireland.

\section{References}

Anstey KJ, Cherbuin N, Budge M, et al. (2011) Body mass index in midlife and late-life as a risk factor for dementia: A meta-analysis of prospective studies. Obesity Reviews 12(5): 426-437. 
Bentler PM and Chou CH (1987) Practical issues in structural modeling. Sociological Methods \& Research 16(1): 78-117.

Berkman L and Syme S (1979) Social networks, host resistance, and mortality: A nine-year follow-up study of Alameda County residents. American Journal of Epidemiology 109(2): 186-204.

Boomsma A (1982) Robustness of LISREL against small sample sizes in factor analysis models. In: Joreskog KG and Wold H (eds) Systems under Indirect Observation: Causality, Structure, Prediction (Part 1). Amsterdam: North-Holland Publishing, pp. 149-173.

Cacioppo JT and Hawkley LC (2009) Perceived social isolation and cognition. Trends in Cognitive Sciences 13(10): 447-454.

Christensen KJ, Moye J, Armson RR, et al. (1992) Health screening and random recruitment for cognitive ageing research. Psychology and Aging 7(2): 204-208.

Cohen S (1988) Psychosocial models of the role of social support in the etiology of physical disease. Health Psychology 7(3): 269-297.

Cohen S, Doyle WJ, Skoner DP, et al. (1997) Social ties and susceptibility to the common cold. Journal of the American Medical Association 277(24): 1940-1944.

Costanzo ES, Lutgendorf SK, Sood AK, et al. (2005) Psychosocial factors and interleukin-6 among women with advanced ovarian cancer. Cancer 104(2): 305-313.

Dantzer R (2001) Cytokine-induced sickness behavior: Mechanisms and implications. Annals of the New York Academy of Sciences 933: 222-234.

DuPertuis LL, Aldwin CM and Bosse R (2001) Does the source of support matter for different health outcomes? Findings from the normative aging study. Journal of Aging and Health 13(4): 494-510.

Eisenberger NI and Cole SW (2012) Social neuroscience and health: Neurophysiological mechanisms linking social ties with physical health. Nature Neuroscience 15(5): 669-674.

Ford ES, Loucks EB and Berkman LF (2006) Social integration and concentrations of C-reactive protein among US adults. Annals of Epidemiology 16(2): 78-84.

Friedman MM (1993) Social support sources and psychological wellbeing in older women with heart disease. Research in Nursing \& Health 16(6): 405-413.

Hannigan C, Coen RF, Lawlor BA, et al. (2015) The NEIL Memory Research Unit: Psychosocial, biological, physiological, and lifestyle factors associated with healthy ageing: Study protocol. BMC Psychology, 3(20): 1-14.

Helminen A, Rankinen T, Vaisanen S, et al. (1997) Social network in relation to plasma fibrinogen. Journal of Biosocial Science 29(2): 129-139.

Holt-Lunstad J, Smith TB, Baker M, et al. (2015) Loneliness and social isolation as risk factors for mortality: A meta-analytic review. Perspectives on Psychological Science 10(2): 227-237.

House JS, Robbins C and Metzner H (1982) The association of social relationships and activities with mortality: Prospective evidence from the Tecumseh Community Health Study. American Journal of Epidemiology 116(1): 123-140.

Hughes S, Jaremka LM, Alfano CM, et al. (2014) Social support predicts inflammation, pain, and depressive symptoms: Longitudinal relationships among breast cancer survivors. Psychoneuroendocrinology 42: 38-44.

Huxhold O, Miche M and Schuz B (2014) Benefits of having friends in older ages: Differential effects of informal social activities on wellbeing in middle aged and older adults. Journals of Gerontology Series B: Psychological Sciences and Social Sciences 69(3): 366-375.

Knox SS and Uvnäs-Moberg K (1998) Social isolation and cardiovascular disease: An atherosclerotic pathway? Psychoneuroendocrinology 23(8): 877-890.

Lee GR and Ishii-Kuntz M (1987) Social interaction, loneliness and emotional wellbeing among the elderly. Research on Aging 9: 459-482.

Li H, Ji Y and Chen T (2014) The roles of different sources of social support on emotional wellbeing among Chinese elderly. PloS ONE 9(3): 90051.

Loucks EB, Berkman LF, Gruenewald TL, et al. (2006) Relation of social integration to inflammatory marker concentrations in men and women 70 to 79 years. American Journal of Cardiology 97(7): 1010-1016.

Lubben J and Gironda M (2004) Measuring social networks and assessing their benefits. In: Phillipson C, Allan G and Morgan D (eds) Social Networks and Social Exclusion: Sociological and Policy Perspective. Boston, MA: Ashgate Publishing.

Marucha PT, Crespin TR, Shelby RA, et al. (2005) TNF- $\alpha$ levels in cancer patients relate to social variables. Brain Behavior and Immunity 19(6): 521-525. 
Nakata A, Irie M and Takahashi M (2014) Sourcespecific social support and circulating inflammatory markers among white-collar employees.Annals of BehavioralMedicine 47(3): 335-346.

Penwell LM and Larkin KT (2009) Social support and risk for cardiovascular disease and cancer: A qualitative review examining the role of inflammatory processes. Health Psychology Review 4(1): 42-55.

Pinquart M and Sorensen S (2001) Influences on loneliness in older adults: A meta-analysis. Basic and Applied Social Psychology 23(4): 245-266.

Radloff L (1977) The CES-D scale: A self report depression scale for research in the general population. Applied Psychological Measurement 1(3): 385-401.

Rosseel Y (2012) lavaan: An R package for structural equation modeling. Journal of Statistical Software 48(2): 1-36.

Runsten S, Korkeila K, Koskenvuo M, et al. (2014) Can social support alleviate inflammation associated with childhood adversities? Nordic Journal of Psychiatry 68(2): 137-144.

Shankar A, McMunn A, Banks J, et al. (2011) Loneliness, social isolation, and behavioral and biological health indicators in older adults. Health Psychology 30(4): 377-385.

Slavich GM and Irwin MR (2014) From stress to inflammation and major depressive disorder: A social signal transduction theory of depression. Psychological Bulletin 140(3): 774-815.

Slavich GM, O'Donovan A, Epel ES, et al. (2010a) Black sheep get the blues: A psychobiological model of social rejection and depression. Neuroscience and Biobehavioral Reviews 35(1): 39-45.
Slavich GM, Way BM, Eisenberger NI, et al. (2010b) Neural sensitivity to social rejection is associated with inflammatory responses to social stress. Proceedings of the National Academy of Sciences of the United States of America 107(33): 14817-14822.

Stranahan AM, Khalil D and Gould E (2006) Social isolation delays the positive effects of running on adult neurogenesis. Nature Neuroscience 9(4): 526-533.

Thoits PA (2011) Mechanisms linking social ties and support to physical and mental health. Journal of Health and Social Behavior 52(2): 145-161.

Tomarken AJ and Waller NG (2003) Potential problems with 'well-fitting' models. Journal of Abnormal Psychology 112(4): 578-598.

Uchino BN, Cacioppo JT and Kiecolt-Glaser JK (1996) The relationship between social support and physiological processes: A review with emphasis on underlying mechanisms and implications for health. Psychological Bulletin 119(3): 488-531.

Weiss R (1974) The provision of social relationships. In: Rubin Z (ed.) Doing Unto Others. Upper Saddle River, NJ: Prentice Hall.

Wolf EJ, Harrington KM, Clark SL, et al. (2013) Sample size requirements for structural equation models: An evaluation of power, bias, and solution propriety. Educational and Psychological Measurement 73(6): 913-934.

$\mathrm{Xu}$ L, Kitade H, Ni Y, et al. (2015) Roles of chemokines and chemokine receptors in obesity-associated insulin resistance and nonalcoholic fatty liver disease. Biomolecules 5(3): 1563-1579.

Yang C, Li T and Frenk SM (2014) Social network ties and inflammation in US adults with cancer. Biodemography and Social Biology 60(1): 21-37. 